\title{
EDITORIAL
}

\section{Clinics Reviews}

\section{Mauricio Rocha e Silva}

Faculdade de Medicina, Universidade de São Paulo - São Paulo/SP, Brazil
This issue of CLINICS which appears as CLINICS: REVIEWS opens a new collection of supplements to be published at a frequency of 1-3 per year. Each supplement will be dedicated to a specific medical chapter. This first issue is dedicated to Neurological and Behavioral Sciences. We believe we have been extremely fortunate in the authors who accepted to cooperate with this new development.

From the Medical Research Council, UK and the Massachusetts Institute of Technology, USA, Timothy Bliss and Sam Cooke have provided us with a highly intriguing discussion on the clinical repercussions of the memory fixation and deletion properties of long-term potentiation and depression. In addition to their physiological relevance, long-term potentiation and depression may have important clinical applications. Molecular mechanisms underlying these processes, and technological advances in non-invasive manipulation of brain activity, now puts us at the threshold of harnessing long-term potentiation and depression, as well as other forms of synaptic, cellular and circuit plasticity to manipulate synaptic strength in the human nervous system. These approaches hold promise for the treatment of a variety of neurological conditions, including neuropathic pain, epilepsy, depression, amblyopia, tinnitus and stroke.

From the Department of Psychiatry of São Paulo University Medical College, Luiz Ferreira and Geraldo Busatto review the very current theme of early diagnosis of Alzheimer's disease. Neuroimaging techniques have provided invaluable information about Alzheimers disease. Due to recent advances, these methods will have an increasingly important role in research and clinical practice. It is now a wellestablished fact that therapeutic strategies will focus on early diagnosis and attempts to retard and ultimately prevent disease evolution. Recent neuroimaging studies of Alzheimers disease have provided relevant information to clinical practice. A new diagnostic modality, i.e. in vivo amyloid imagingis proving to be a valuable addition to the more established procedures of magnetic resonance, single photon emission computed tomography(SPECT) and 18F-fluorodeoxyglucosepositron emission tomography (FDG-PET).

From the Department of Neurology of Duke University the Research Group headed by Miguel Nicolelis provides us with a review on future developments in brain machine interface research by Mikhai Lebedev et al. Neuroprosthetic devices based on brain-machine interface technology hold

Email: mrsilva36@hcnet.usp.br

Tel.: 5511 3069-6235

Copyright (c) 2011 CLINICS - This is an Open Access article distributed under the terms of the Creative Commons Attribution Non-Commercial License (http:// creativecommons.org/licenses/by-nc/3.0/) which permits unrestricted noncommercial use, distribution, and reproduction in any medium, provided the original work is properly cited. promise for the restoration of body mobility in patients suffering from devastating motor deficits caused by brain injury, neurological diseases and limb loss. Progress has been recently achieved in this multidisciplinary research, mainly in brain-machine interactors that enact upper-limb functionality, but many problems need to be resolved before fully functional limb neuroprostheses can be built. This paper reviews future steps that are part of the strategic plan of Duke University and its partners to bring this new technology to clinical fruition.

Suely Marie and Sueli Shinjo, from the Department of Neurology of São Paulo University Medical College brings us new insights into the interaction of cellular energy metabolism and brain cancer, in which they review current literature and present original new data. A crucial point made here is that to support rapid proliferation, cancer cells select glycolysis in the presence of oxygen (Warburg effect) to fuel macromolecules for the synthesis of nucleotides, fatty acids, and aminoacids for the accelerated mitosis, rather than fuel the tricarboxylic acid cycle and oxidative phosphorylation. Metabolic pathways are modified by cancer cells and the interactions between oncogenes and tumor suppressor genes with these pathways may enlighten new strategies for cancer therapy. Potential therapeutic targets of the cancer energetic metabolism are enumerated, highlighting the astrocytomas, the most common brain cancer.

The pathophysiology of Alzheimer's disease is discussed from the same Department of Psychiatry of São Paulo University by Evelin L. Schaeffer, Micheli Figueiró and Wagner F. Gattaz who describe the main transgenic mouse models of Alzheimer's disease which have been adopted in Alzheimer's research, and discuss the insights into its pathogenesis from studies in such models. These have been key to the understanding of the roles of soluble $\beta$ amiloidoligomers in disease pathogenesis, as well as of the relationship between pathologies relating $\beta$-amiloidplaques or to neurofibrillary tangles containing hyperphosphorylated Tau protein.

Carla Scorza and Esper Cavalheiro from the Department of Neurology of the Federal University of São Paulo review the significant theme of translational research relating to animal models of animal intelligence disabilities. Human intellectual disability affects $2-3 \%$ of the general population, making it a daunting, but poorly understood social problem characterized by limitations in intellectual functioning and in adaptive behavior. Studies in humans have a very limited capacity to explain basic mechanisms of this condition, making animal models an invaluable investigative tool. Translational research specifically aims at taking basic scientific discoveries and best practices to benefit the lives of people and this review describes animal models of potential risk factors for intellectual disabilities. 
From the same Department, Fulvio Scorza et al. review a much neglected issue: sudden unexpected death in epilepsy. This is one of the most common neurologic problems worldwide. Unfortunately, individuals with epilepsy are at higher risk of death than the general population, and sudden unexpected death in epilepsy is the most important direct epilepsy-related cause of death. This article focuses on the risk factors, mechanisms and preventative measures obtained from clinical and experimental studies on sudden unexpected death in epilepsy.

Peyman Yeganeh-Doost et al., from the Departments of Psychiatry, University of Göttingen and University of São Paulo provide us with a provoking review on the role of the cerebellum in schizophrenia. The cerebellum is involved in cognitive functions such as attention, working memory, verbal learning and sensory discrimination. In schizophrenia, a disturbed prefronto-thalamo-cerebellar circuit has been proposed to play a role in the pathophysiology. A deficit of the glutamatergic NMDA receptor has been hypothezised. The risk gene neuregulin 1 (NRG-1) may play major role in this process. Altered expressions of receptors in right cerebellar regions of schizophrenia patients, which may be secondary upregulations due to dysfunctional receptor has been shown. A dysfunction of the gamma amino butyric system may also be involved. Molecular findings in the light of the role of the cerebellum in attention and cognitive deficits in schizophrenia are discussed. 